\section{JAW DROPPING NEW TREATMENT FOR TMJ LAUNCHES}

A revolutionary new way to treat temporo-mandibular joint disorders has been approved for use in the UK and Ireland. The Cerezen device comprises a pair of removable custom-made hollow inserts which are placed within the ear canal to reduce the symptoms of temporo-mandibular joint disorders (TMJD), pain and related conditions, including grinding of teeth and headaches.

Simply inserted in the ear, the nearest access point to the temporo-mandibular joint, the device works by exerting subtle pressure on the walls of the ear canal when the jaw is in the closed position. This encourages the patient to return to the 'open bite' position, minimising the tendency to clench the jaw and tense the surrounding muscles.

The device is quick and simple to fit, requiring only around 15 minutes' chair time plus 25 minutes for ear impression by a trained professional such as an audiologist. Once the diagnosis is made and ear impressions taken and submitted, TMJ Health LLC produces the custom devices and supplies them in approximately two weeks. They are easy and convenient for the patient to insert and remove, offering a simple and safe reduction of TMJD symptoms without impacting the patient's lifestyle, as speech, eating and drinking are unaffected.

As the device is hollow, it does not affect hearing, and is fitted with small retraction posts to make it easy to remove - which is advised for bathing and swimming.

Following regulatory approval, the Cerezen device will be available in the UK from May 2015.

For more information about the device visit www.cerezen.eu.

\title{
FAST AND OBJECTIVE PREPARATION EVALUATION
}

The KaVo Dental Teacher is a new system for fast and objective tooth preparation validation. With the additional teaching modules 'Virtual wax-up' and 'Virtual articulation' it moreover offers tutors tools to effectively teach complex contents such as articulation and wax-up procedures. The Dental Teacher System includes a 3D scanner, PC and three software modules.

The KaVo Dental Teacher offers the quickest way to evaluate student preparations. The whole process just takes approximately two minutes. It offers an objective, automatic and computer-based workflow which relieves tutors of validating numerous in-between steps of many student's preparations and supports them to concentrate on core tasks. Through its 3D, colour-graded visualisation of the comparison between student's and master's preparation Dental Teacher enables students to better understand the deviations between their preparation and the ideal preparation defined by the lecturer. Dental Teacher ensures objective, and constant results and correct documentation of students' work during study courses as well as during examinations.

Teaching occlusion, articulation and functional jaw movements can be frustrating when using only pictures and papers. The KaVo Virtual Articulator visualises all occlusal parts therefore making it better understandable for students. The students learn easily, because they can see how each section of the tooth morphology interacts with its antagonist during the movement.

The teaching module 'Virtual Wax-up' is great for learning how to wax-up artificial dentition for pre-clinic and patient treatment. Furthermore, it offers perfect conditions to train the use of CADCAM technology from the very beginning.

\section{CPD PROGRAMME CONTINUES TO SUPPORT DENTAL HEALTHCARE PROFESSIONALS}

Johnson \& Johnson, the makers of LISTERINE, are delighted to bring you new content following the successful introduction of our CPD programme in 2014, with the aim of supporting the ongoing CPD needs of dental healthcare professionals in improving and maintaining the oral health of their patients.

Through our programme, we aim to deliver recent clinical findings, useful information for in-practice management of oral diseases and patient communication strategies for improved preventive home care, as well considering the effects of lifestyle and other external influences on your patients' oral health.

Visit www.listerineprofessional.co.uk/cpd-

educational-programme to earn verifiable hours from our expanding library, including our newest pieces on the challenge of dental anxiety, gum health: a systematic review update and meeting the oral health need of an ageing population.

In addition, soon to be made available are CPD articles on successfully delegating care and healthrelated technology in the dental care setting.

Upon completing each module successfully, we will send you a CPD certificate via email within 14 days.

For more information, email dentalcpd@its.jnj.com.

\section{WHICH MASSAGE IS RIGHT FOR YOU?}

The team at Physio Comes To You think that it is crucial to know what massage is best for your needs.

That is why we have put together a simple and easy guide to finding the right massage for you.

People look for different things from their massage. From pain or injury relief to relaxation, health and wellbeing, everybody is different. Our flow chart walks you through the bet options for you. Choose from aromatherapy, Swedish, sports, deep tissue, Thai, Shiatsu, pregnancy, hot stone and trigger point massages to get you back on your feet again. We cover London and are now extending Physio Comes To You nationwide.

To find a massage that is right for you visit www. physiocomestoyou.co.uk or call 02078840374 or 03302233407 for outside London.

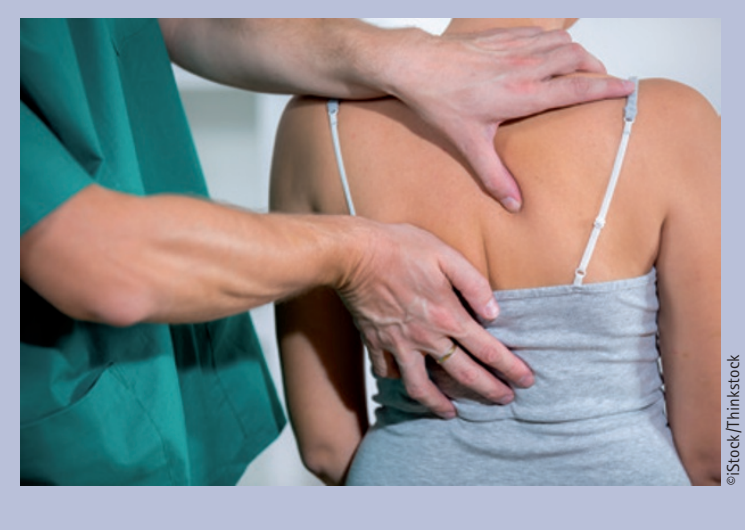

